\title{
FREQUENCY DOMAIN EQUALIZER FOR MULTIRATE UMTS-TDD SYSTEMS
}

\author{
Luis Gonçalves, Atilio Gameiro \\ Instituto de Telecomunicações, Campus Universitário de Santiago, 3810-193 Aveiro, Portugal \\ Departamento de Electrónica e Telecomunicações, Universidade de Aveiro, Portugal \\ email: lgoncalves@av.it.pt,amg@det.ua.pt
}

\begin{abstract}
Direct Sequence Spread Spectrum (DS-SS) signals exhibit cyclostationary properties which imply a redundancy between frequency components separated by multiples of the symbol rate. In this paper we present a Multiple Access Interference Canceller that explores this property and applies to UMTS-TDD. This linear frequency domain canceller operates in the spreaded signal in such way that the interference and noise at its output is minimized (Minimum Mean Squared Error Criterium). The performance is evaluated in two configurations: one including the Frequency Shift Canceller (FSC) and the other concatenated with Parallel Interference Canceller (PIC). The results are benchmarked against the performance of the conventional RAKE detector and the conventional PIC detector.
\end{abstract}

Index Terms - cyclostationary, redundant, frequency shift, PIC, DS-CDMA

\section{INTRODUCTION}

Direct sequence spread-spectrum (DS-SS) code division multiple access (CDMA) has emerged as one of the most promising techniques to implement various radio communication systems. It presents significant advantages over Time Division Multiple Access (TDMA), namely frequency diversity, multipath diversity and more spectrum efficiency on multicell systems [1], which led to its choice as the technology for third generation cellular systems. The first version of third generation CDMA systems will be based on the conventional RAKE receiver, which is known to be limited by the multiple access interference (MAI) and require a very precise power control. To overcome these limitations and therefore enhance the capacity of CDMA systems, joint detection of the received DS-SS signals has been proposed to be used at the base station (BS) or at the user equipment. The optimum joint detector [2] although well known requires however a prohibitively high computational complexity, and consequently effort has been made to devise suboptimum algorithms with good compromise between performance and complexity that can be implemented without prohibitive costs in near future CDMA systems. This communication fits in this approach, and aims at presenting a moderate complexity MAI canceller operating on the broadband DS signal. The Frequency Shift Detector can be used either as standalone unit or it can be used prior to a PIC where it is intended to produce signals clean enough so that the first decisions of the PIC can be considered reliable enough to be used by the subsequent stages.

This work is being supported by a PhD Grant from PRAXIS XXI, Fundacão para a Ciência e Tecnologia and the projects ASILUM and VISEF
The DS-SS signal is a particular case of a stationary random pulse amplitude modulation. This kind of signals are known to have cyclostationary properties [3], [4]. Those properties imply redundancy between frequency components separated by multiples of the symbol rate. It is this characteristic that we explore to propose a new MAI canceller.

In previous work [5] the Frequency Shift Canceller (FSC) was defined and implemented for a fixed spreading factor. The results have shown that considerable improvement is achieved against the RAKE and the FSC even outperforms the PIC for moderate to high values of $E_{b} / N_{0}$. The FSC was also tested as a precanceller to be used prior to a PIC and the results have shown that nearly complete interference cancellation in a UMTS-TDD scenario with up to eight simultaneous users with spreading factor in the same slot.

In the present paper the FSC was implemented to comply with full UMTS-TDD norms. This enables the transmission of services with different rates by varying the spreading factor, but the structure of the spreading which is a composite operation involving channelization and scrambling, in UMTS-TDD, leads to codes that extend over several symbols.

The paper is outlined as follows. In section two we show that in a DS signal non overlapping frequency bands separated by a multiple of the baud rate are linearly related. This result is used to present in section three the architecture and design principles of a MAI canceller that explores this redundancy. In section four we present simulation results that illustrate the performance provided by the new canceller. Finally in section five the main conclusions of this work are outlined.

\section{THEORETICAL BACKGROUND}

A DS-SS signal with spreading factor $Q_{\max }$ (maximum spreading factor allowed in the system) is represented by

$$
s(t)=\sum_{k} a_{k} g(t-k T)
$$

where $\left\{a_{k}\right\}$ is the sequence of information symbols, $\frac{1}{T}$ the symbol rate and $g(t)$ is the signature waveform.

The Fourier Transform of this signal is

$$
S(f)=\sum_{k} a_{k} G(f) e^{-j 2 \pi f k T}=G(f) A(f)
$$

with

$$
A(f)=\sum_{k} a_{k} e^{-j 2 \pi f k T}
$$




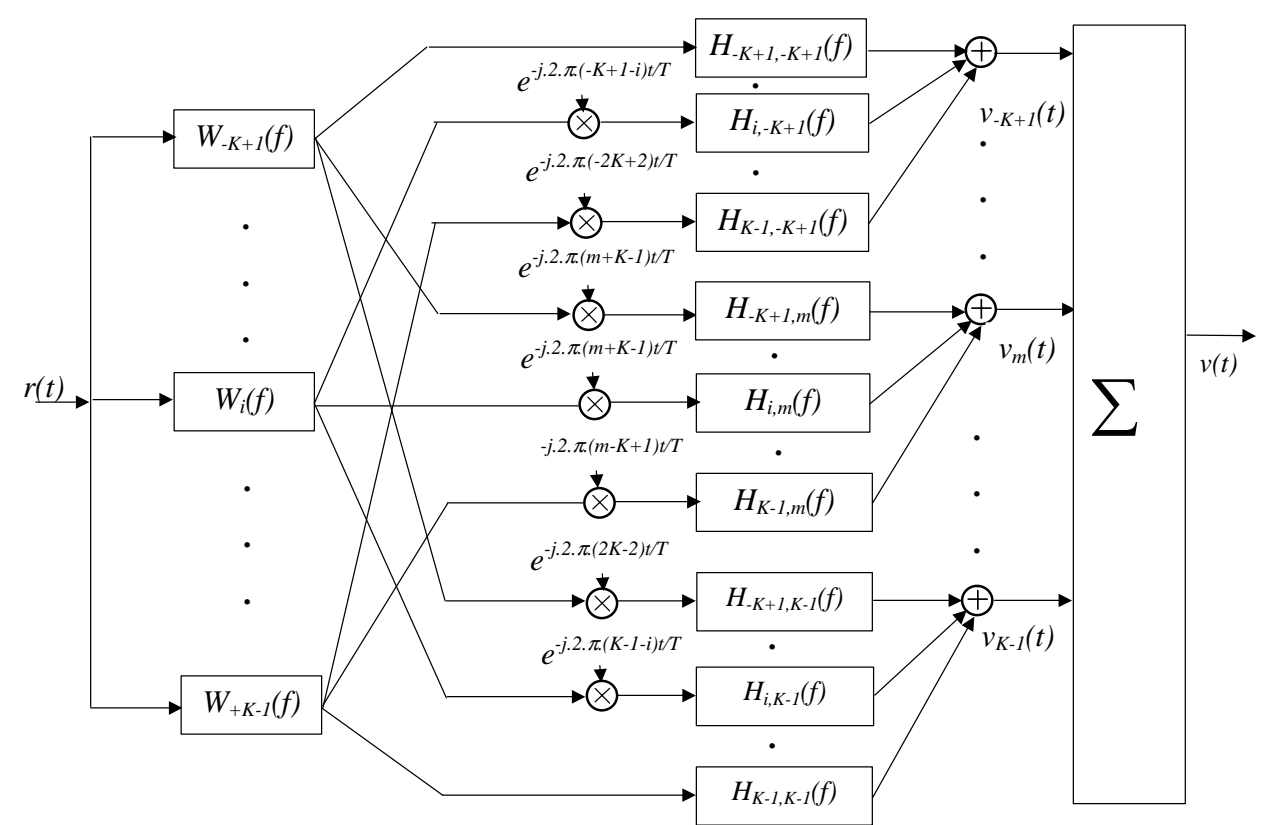

Fig. 1. Conceptual Schematic of the Canceller

From (3) it is easy to verify that

$$
A\left(f+\frac{i}{T}\right)=A(f) \forall i \in \mathrm{Z}
$$

Let us define

$$
S_{m B}(f)=S\left(f+\frac{m}{T}\right) \operatorname{rect}(f T)
$$

and

$$
G_{m B}(f)=G\left(f+\frac{m}{T}\right) \operatorname{rect}(f T)
$$

where $m$ is the index of the band.

Then using (2)

$$
S_{m B}(f)=G_{m B}(f) A(f)
$$

Let $m=m_{1}$ and $m=m_{2}$ (representing two bands)

$$
S_{m_{1} B}(f)=G_{m_{1} B}(f) A(f)
$$

and

$$
S_{m_{2} B}(f)=G_{m_{2} B}(f) A(f)
$$

Therefore assuming $G_{m_{1} B}(f)$ has no singularities, we can conclude that in the interval $f \in\left[0, \frac{1}{T}\right.$ [ is verified that

$$
S_{m_{2} B}(f)=\frac{G_{m_{2} B}(f)}{G_{m_{1} B}(f)} S_{m_{1} B}(f)
$$

This means that we can relate the signal information in nonoverlapping frequency bands spaced by a multiple of the baud rate by a linear transformation.

\section{PRinciples OF THE CANCELleR}

The canceller operates in the frequency domain and in a practical implementation or in a simulation system the time to frequency domain conversion is performed digitally through a Fast Fourier Transform (FFT). In the following derivation we adopt however a continuous signal notation.
The architecture of the canceller is shown in figure 1 , for a given user. In a base station where all the signals have to be recovered the canceller consists of the replica of this basic receiver for each user.

The input signal $r(t)$ in figure 1 is defined as $\sum_{u=1}^{U} s^{(u)}(t)+$ $n(t)$ where $\mathrm{U}$ is the number of users and $n(t)$ is stationary noise with power spectral density $\eta_{i n}(f)$. We consider without loss of generality that user one is the user of interest. The objective and design criteria for the canceller is to minimize the overall disturbance (MAI+noise) subject to the condition that $s^{(1)}(t)$ is not distorted. This constraint implies that the filters in figure 1 which convert the band $i$ to band $m$ (see section II) be of the form

$$
H_{i, m}(f) \propto X_{i, m}(f)=\frac{G_{m B}^{(1)}\left(f-\frac{m}{T}\right)}{G_{i B}^{(1)}\left(f-\frac{m}{T}\right)}
$$

where $G_{m B}^{(1)}(f)$ is defined as

$$
G_{m B}^{(1)}(f)=\operatorname{rect}(f T) G^{(1)}\left(f+\frac{m}{T}\right)
$$

with $G^{(1)}(f)$ being the Fourier transform of $g^{(1)}(t)$ which is the signature waveform of $s^{(1)}(t)$.

In those conditions the band $m$ of the signal at the output is

$$
\begin{aligned}
v_{m}(t) & =s_{m}^{(1)}(t)\left(\sum_{i} \alpha_{i m}\right) \\
& +\sum_{u=2}^{U}\left[\sum_{k} a_{k}^{(u)} \beta_{m}^{(u)}(t-k T)\right]+n_{m}^{\prime}(t)
\end{aligned}
$$

where

$$
\begin{aligned}
\beta_{m}^{(u)}(f) & =\sum_{i} \alpha_{i m} X_{i, m}(f) G_{i B}^{(u)}\left(f-\frac{m}{T}\right) \\
& =\sum_{i} H_{i, m}(f) G_{i B}^{(u)}\left(f-\frac{m}{T}\right)
\end{aligned}
$$


, The power spectral density of additive the noise disturbance $n_{m}^{\prime}(t)$ is

$$
\begin{aligned}
\eta_{\text {out }_{m}}(f) & =\sum_{i}\left|\alpha_{i m}\right|^{2}\left|X_{i, m}(f)\right|^{2} \eta_{i n_{i B}}\left(f-\frac{m}{T}\right) \\
& =\sum_{i}\left|H_{i, m}(f)\right|^{2} \eta_{i n_{i B}}\left(f-\frac{m}{T}\right)
\end{aligned}
$$

where $\eta_{i n_{i B}}(f)$ is defined as:

$$
\eta_{i n_{i B}}(f)=\operatorname{rect}(f T) \eta_{i n}\left(f+\frac{i}{T}\right)
$$

The design criteria implies that the weights $\alpha_{i m}$ are dimensioned so that the Minimum Squared Error (MSE)

$$
\sum_{u=2}^{U}\left[\sum_{k}\left|a_{k}^{(u)}\right|^{2} \int_{f}\left|\beta_{m}^{(u)}(f)\right|^{2} d f\right]+T_{B t} \int_{f} \eta_{\text {out }_{m}}(f) d f
$$

is minimized subject to the condition that $\sum_{i} \alpha_{i m}=1$. In (17) $T_{B t}$ is the burst duration time and the symbols $a_{k}$ considered correspond to the ones existing in this burst.

Each band of the output signal $v(t)$ depends linearly of the input bands with bandwidth $\frac{1}{T}$ and spaced by multiples of $\frac{1}{T}$. The performance of the interference canceller could be enhanced if we divide the input and output bands in subbands of equal bandwidth. Then each output subband depends linearly of the input subbands with the same bandwidth and spaced by multiples of $\frac{1}{T}$ (symbol rate) of the output subband. The shorter the subband better the performance.

The UMTS-TDD standard was designed to accommodate several simultaneous transmission rates. The different rates are accomplished by varying the spreading factor. The spreading code is composed by the product between the channelization code and the scrambling code. The channelization code lasts for one symbol and its number of chips is equal to the spreading factor. The scrambling code lasts for sixteen chips (the maximum spreading factor $Q_{\max }$ ) or during $\frac{Q_{\max }}{Q}$ symbols being $Q$ the spreading factor. Then to construct the spreading code the channelization code must be repeated $\frac{Q_{\max }}{Q}$ and multiplied by the scrambling code. The spreading code extends for more than one symbol in the case of $Q \neq Q_{\max }$. In the case of UMTS-TDD, $Q_{\max }=16$.

The approach to extend the canceller explained above to multirate is to decompose each signal with a spreading factor different of sixteen in several signals with spreading factor of sixteen.

The representation in the time domain of a generic user is

$$
s(t)=\sum_{k} \sum_{l=0}^{\frac{Q_{\max }}{Q}-1} a_{k}^{l} g_{l}\left(t-l T-\frac{Q_{\max }}{Q} k T\right)
$$

where $\left\{a_{k}^{l}\right\}$ are the sequences of information symbols, $\frac{1}{T}$ the symbol rate (depends of the spreading factor) and $g_{l}(t)$ are the components of signature waveform. To obtain these components the spreading code (length equal to $Q_{\max }$ ) is divided in $\frac{Q_{\max }}{Q}$ sequences of length $Q$ and then each one is pulse shaped (Raised Cosine) and affected by channel estimates.
This signal is then divided in $Q_{\max } / Q$ signals

$$
s_{l}(t)=\sum_{k} a_{k}^{l} g_{l}\left(t-l T-\frac{Q_{\max }}{Q} k T\right)
$$

with $l \in\left\{0,1 \ldots, Q_{\max } / Q-1\right\}$ having each a signature waveform $g_{l}(t-l T)$.

The canceller is applied to same input signal $r(t)$ which is the sum of all the signals. Only the signatures waveforms used in the processing are the ones of the new users of spreading factor of sixteen.

Some of the FFT of the signatures waveforms of (19) have zeros at some frequencies giving filters in (11) with infinite response. For that cases is not made a minimization with (17) but it is forced the FFT of the output of the FSC to zero in those frequencies.

\section{Application of the CANCELler to UMTS-TDD}

In this section we present some numerical results illustrating the performance of the proposed detector configurations with UMTS-TDD signals. The proposed configurations are more suitable to be implemented in the uplink because the detectors require knowledge of the spreading codes of the active users. To evaluate the canceller performance a simulation chain was implemented. Basically this simulation chain is composed by a transmitter, a transmission channel and a receiver.

\section{A. Transmitters}

The transmitters are compliant with the 3GPP specifications for UMTS-TDD.

\section{B. Channel Model}

The channel model used in this work was the Geometrical Based Single Bounce Elliptical Model (GBSBEM) proposed by Liberti [6]. This model was developed for microcell and picocell environments. The propagation channel is characterized by $L$ paths for each user, one in line of sight plus $L-1$ arriving from remote reflectors located randomly within an ellipsis where the base station and the mobile unit are at the foci. Each path is characterized by complex constant and a delay. The delay is uniformly distributed between zero and the maximum delay spread. The phase of the complex constant

TABLE I

SIMULATION PARAMETERS SETTINGS

\begin{tabular}{|l|c|}
\hline Spreading Factor & $16,8,4$ \\
\hline Number of Taps & 2 \\
\hline Channel & GBSBEM \\
\hline Velocity & $50 \mathrm{Km} / \mathrm{h}$ \\
\hline Path Loss & 3.7 \\
\hline BurstType & 1 \\
\hline Maximum Delay Spread & $2.0 \mu \mathrm{s}$ \\
\hline Degrees of Freedom of FSC & 16 \\
\hline Number of samples per chip & 4 \\
\hline Line of Sight Distance & $300 \mathrm{~m}$ \\
\hline
\end{tabular}




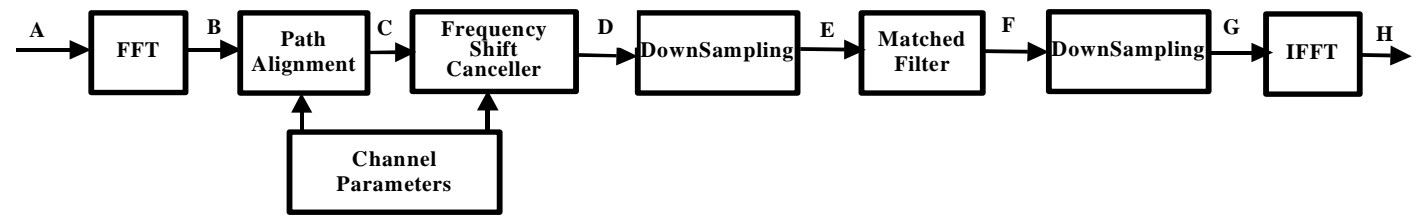

Fig. 2. Receiver with FSC

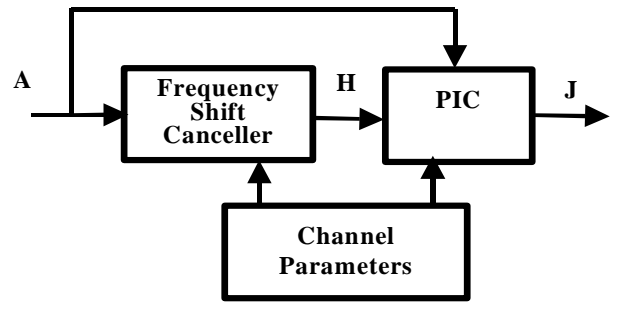

Fig. 3. Receiver including FSC plus PIC

is uniformly distributed in $[0,2 \pi[$. The channel model used takes in account the Doppler Effect. The amplitude of the complex constant follows a Rayleigh distribution. The channel parameters are assumed to be constant within each burst.

\section{Receivers}

Figure 2 depicts the basic configuration for the detector that includes the Frequency Shift Canceller. If we remove the Frequency Shift Canceller block the detector is a conventional RAKE. The Path Alignment and Downsampling are blocks whose operations are done in frequency domain despite the fact that the names reflect the correspondent time domain operations. The path alignment includes delay alignment and maximum ratio combining of the spreaded signal. The signal in $A$ (figure 2) has a resolution of four samples per chip and the first downsampling has the same factor. The second downsampling takes a factor equal to the spreading factor. In $H$ we have the signal before symbol decision. Notice that the Discrete Fourier Transform is made only once in each slot (and burst).

The second detector configuration to be evaluated is the detector composed by FSC plus PIC (figure 3). The FSC in figure 3 corresponds to the whole receiver chain of figure 2 . The FSC provides the signal for the symbol decision inside the PIC.

These two configurations are benchmarked with the conventional RAKE and conventional single stage PIC.

\section{Results}

The simulations were made with the parameters shown in Table I. The channel parameters estimates were assumed to be perfect.

Following we present some results for the scenario.

- Scenario:

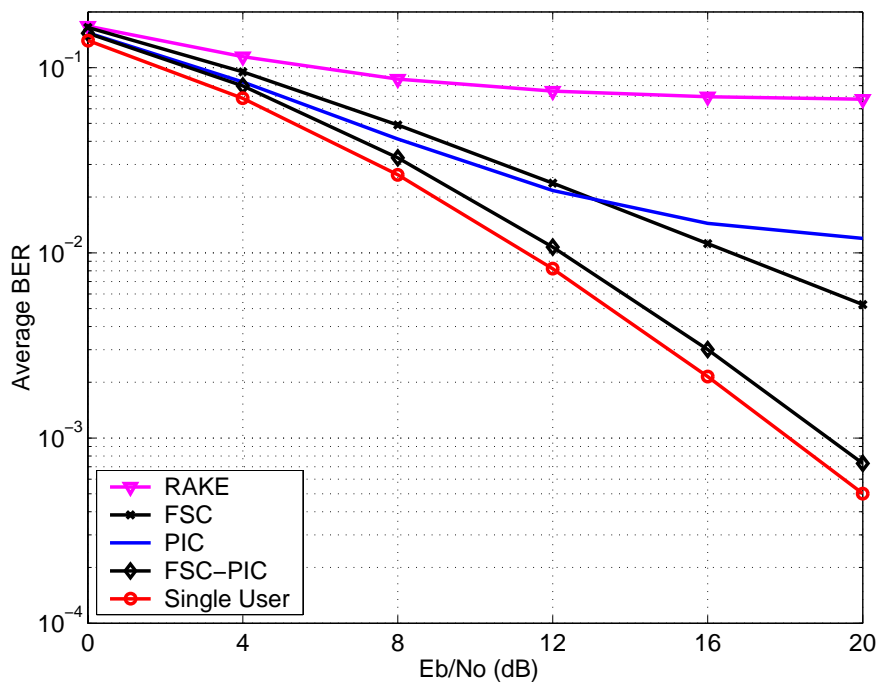

Fig. 4. BER for users with spreading factor of sixteen

- Eight users: Four with spreading factor of sixteen, two with spreading factor of eight and two with spreading factor of four (Full load system).

- The users with spreading factor eight and four have powers above the users of spreading factors of sixteen of 3 and $6 \mathrm{~dB}$ respectively.

Plots of the BER achieved are shown in figures 4-6 and apply to users with spreading factors of 16,8 and 4 respectively.

In each plot we compare the following configurations: RAKE, PIC, FSC, FSC+PIC and Single User. The BER shown for a given spreading factor is the average of the various users with the same spreading factor.

The results show that significant gains are obtained by the FSC and FSC+PIC over the RAKE receiver. Even it is reached almost single user performance with FSC+PIC detector in all spreading factors. For all spreading factors the FSC outperforms the PIC for high $E_{b} / N_{0}$.

Relatively to the algorithmic complexity, and comparing the FSC against the time domain MMSE (see [7]) the FSC requires more matrix inversions but with smaller size matrices $(15 \times 15$ with UMTS-TDD). The resulting matrices are hermitic semidefinite positive and the method used for inverting them is Cholesky Decomposition. The main complexity of the canceller is not due to the inversion of the matrices (which are small sized) but to the calculus of elements of these matrices. Comparing the FSC using these referred methods against the time domain MMSE [7] we find out that the FSC is about tree times less complex, when considering 16 simultaneous users with a 


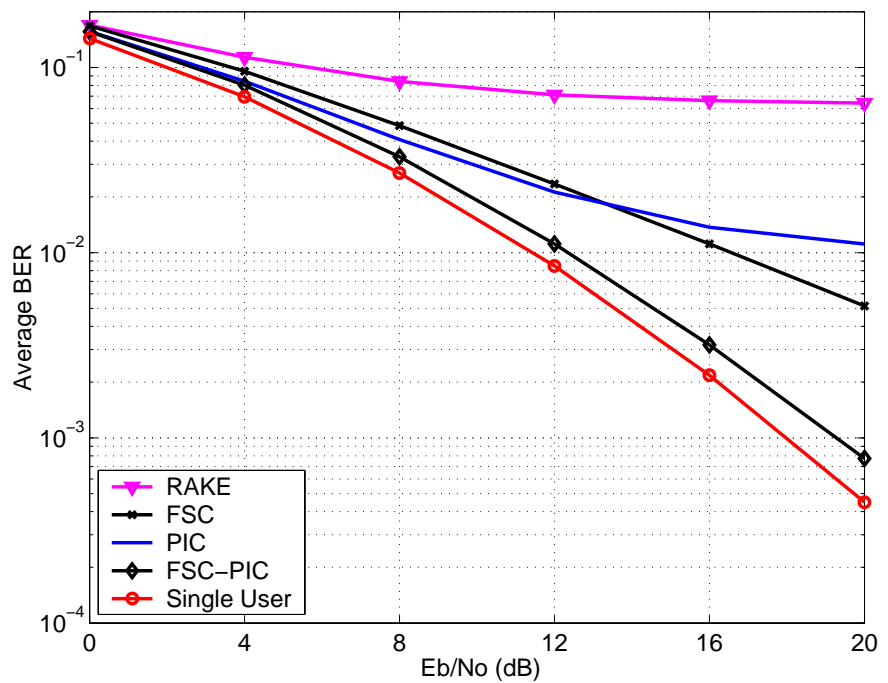

Fig. 5. BER for users with spreading factor of eight

spreading factor of sixteen, one burst and an exact Cholesky Decomposition. Relatively to the PIC the FSC is more complex but the FSC+PIC Combination achieves a better performance than a two stage PIC.

\section{CONClusions}

In this communication we presented a new linear multirate canceller operating in the frequency domain that takes advantage of frequency redundancy of the DS-SS signals. The results show that significant gains are obtained by the FSC and FSC+PIC over the RAKE receiver. Even it is reached almost single user performance with FSC+PIC detector in all spreading factors. For all spreading factors the FSC outperforms the PIC for high $E_{b} / N_{0}$.

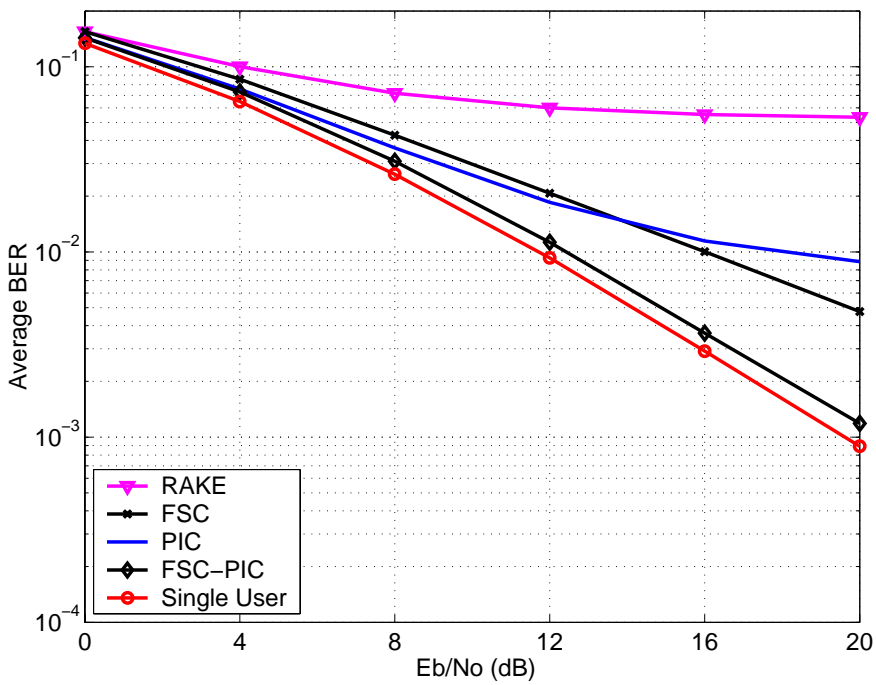

Fig. 6. BER for users with spreading factor of four

\section{REFERENCES}

[1] Ryuji Kohno, Reuven Meidan and Laurence B. Milstein, Spread Spectrum Access Methods for Wireless Communications, IEEE Communications Magazine, January 1995

[2] Sergio Verdu, Minimum Probability of Error for Asynchronous Gaussian Multiple-Access Channels, IEEE Transactions of Information Theory, January 1986

[3] Gardner, William A., Cyclostationarity in Communications and Signal Processing, IEEE PRESS, 1994

[4] Gardner, William A., Exploitation of Spectral Redundancy in Cyclostationary Signals, IEEE Signal Processing Magazine, April 1991

[5] Luis Gonçalves, Adão Silva, Atilio Gameiro, Frequency Shift Based Multiple Access Interference Canceller for DS-CDMA Systems, Vehicular Technology Conference 2001 Fall, October 2001, Atlantic City, USA.

[6] J. C. Liberti, T. S. Rappaport, Smart Antennas for Wireless Communications: IS-95 and Third Generation CDMA Applications, Prentice Hall, 1999.

[7] Marius Vollmer, Martin Haardt and Jürgen Götze, Comparative Study of Joint-Detection Techniques for TD-CDMA Based Mobile Radio Systems, IEEE Journal on Selected Areas in Communications, August 2001. 